\title{
OCCURRENCE OF VESICULAR ARBUSCULAR MYCORRHIZAL FUNGI IN TROPICAL FOREST COMMUNITIES OF INDIA
}

\author{
TRIPATHI, P.* - KHARE, P.K. \\ Department of Botany, Dr. H.S. Gour Central University, \\ Sagar (M.P.) 470003, India \\ *Corresponding author \\ e-mail: priyanganaeco@gmail.com \\ (Received $16^{\text {th }}$ November 2009; accepted $5^{\text {th }}$ January 2012)
}

\begin{abstract}
The paper deals with the quantitative and qualitative assessment of vesicular arbuscular mycorrhizal fungi (AMF) associated with the rhizosphere of dominant plants of three vegetation viz., Tropical Evergreen Forest, Gibbon Wild-life sanctuary, Jorhat, Assam, Tropical Moist Forest, Kanha National Park, Mandla, M.P., and Tropical Dry Deciduous Forest, Sagar, M.P. Root and rhizosphere soil samples, collected near different trees species were analyzed for root colonization, spore count, extramatrical hyphae, vesicles, Paris coil and intraradical spores. VAM spores were isolated by wet sieving and decanting method and estimation of spores was carried out. In evergreen forest vegetation (Site-I), maximum root colonization $(33 \pm 0.35 \%)$ was observed in Terminalia myrocarpa Henrck \& Mull. and minimum in Walsura robusta Roxb. (19 $\pm 0.61 \%)$.VAM spore count per $25 \mathrm{~g}$ of soil was also highest (133 \pm 0.41$)$ in Terminalia myrocarpa Henrck \&Mull. while it was low in Mesua ferrea Linn. (48 \pm 0.21 ). In tropical moist forest (Site-II), Bridelia retusa Spreng. was observed to support least root colonization ( $33 \pm 0.4$ per cent) and spore density $(43 \pm 0.25 / 25 \mathrm{~g}$ soil). Maximum root colonization ( $64 \pm$ $0.49 \%)$ and spores density $(167 \pm 0.46)$ were found in Lagerastroemia parviflora Roxb. In tropical dry deciduous forest (Site-III), root colonization $(62 \pm 0.62 \%)$ and spores density $(121 \pm 0.31)$ were maximum in Albizzia lebbek Benth.-highest among all the three sites. Interestingly, these attributes were also lowest at this site in Wrightia tinctoria Linn. In all, 25 VAM species were identified from all the three forest sites. Out of these, 5 VAM species were common to three forest sites. More number of exclusive VAM species (6) were recorded at site-II. Glomus spp. was found as dominant VAM at all the three forest sites. Besides, the other morphological details of different VAM fungi, the results of present investigation envisage that diverse conditions of the habitat i.e. Tropical Moist Forest, support more VAM species not only in terms of numbers but also as the root association and potential propagules in the form of spores.
\end{abstract}

Keywords: VAM, Root colonization, Qualitative and Quantitative characters

\section{Introduction}

The Vesicular Arbuscular Mycorrhizal (VAM) association with most of the terrestrial plants is perhaps as old as the evolution of terrestrial flora (Taylor et al., 1995). They are ubiquitous in distribution from tropical (Chaurasia and Khare, 2005), temperate (Vestberg, 1995) and arctic (Dalpe and Aiken, 1998) regions. They are responsible for enhancing the growth and improving the health of plants and soils (Hodge et al., 2001; Rausch et al., 2001) and play a crucial role in mineral nutrition of forest trees thus becoming important nutrient acquiring mechanisms (Pate, 1994).

Although the occurrence of VAM species is non specific, they exhibit variation in both qualitative and quantitative attributes. Variation may be found due to climatic, edaphic and composition of vegetation types.

The present paper deals with the occurrence and population variations of arbuscular mycorrhizal fungi in three different tropical forests of India namely tropical evergreen forest, tropical moist deciduous forest and tropical dry deciduous forest. Root 
colonization, spore population and qualitative characteristics like external hyphae, vesicle, Paris coil, intraradical spores were determined and discussed.

\section{Study area}

Site-I The site belongs to a tropical evergreen forest within Gibbon National Park near Jorhat, Assam, about $30 \mathrm{~km}$ West of Jorhat, situated at $26^{\circ} 46^{\prime} \mathrm{N}$ latitude and $96^{\circ} 16^{\prime} \mathrm{E}$ longitude Climate is a seasonal with average monthly minimum and maximum temperature of $19^{\circ} \mathrm{C}$ and $30^{\circ} \mathrm{C}$ in January and May respectively. Annual rainfall is more than $300 \mathrm{~cm}$ received throughout the year. The soil is clay to sandy clay with $\mathrm{pH}$ ranging from 4.3 to 4.9. The forest types as classified by (Champion and Seth, 1968) is semievergreen to evergreen. Dominant forest tree species are Dipterocarpus myrocarpa Vesque, Walsura robusta Roxb., Dillenia indica Linn.

Site-II The site, tropical moist forest, is a part of Kanha National Park, a protected area dedicated to Project Tiger in the state of Madhya Pradesh in Central India. It is located in between $22^{\circ} 1^{\prime}$ and $22^{\circ} 27^{\prime} \mathrm{N}$, and $80^{\circ} 26^{\prime}$ and $81^{\circ} 4^{\prime} \mathrm{E}$ longitude at an altitude of 800-900 msl. The climate is distinctly seasonal with three well marked reason viz., rainy, winter and summer. Maximum temperature of $40^{\circ} \mathrm{C}$ reaches in summer and minimum as $0.0^{\circ} \mathrm{C}$ during the month of December in winter season. The total annual rainfall is $140 \mathrm{~cm}$ principally received during the months of July to September. Edaphically it constitutes the Central Indian highlands that forms main peninsula of India. The terrain is undulating with clayey laterite rich in Bauxite on tops and plains, and valley with granite gneisses. The soil is near neutral or alkaline type. Forests are tropical moist deciduous types dominated chiefly by Shorea robusta Gaertner F.

Site-III This site is a part of tropical dry deciduous forests in Central India, located in between $23^{\circ} 5^{\prime}-24^{\circ} 25^{\prime} \mathrm{N}$ and $78^{\circ} 10^{\prime}-79^{\circ} 15^{\prime} \mathrm{E}$ longitude at an elevation of $583 \mathrm{msl}$ near the town of Sagar in the state of Madhya Pradesh. The climate is distinctly seasonal with summer, rainy and winter seasons. Total annual rainfall is $120 \mathrm{~cm}$, nearly $90 \%$ of which is received in rainy season. Summer is hot with maximum temperature touching $47^{\circ} \mathrm{C}$ and winter is pleasant with minimum temperature of $8-9^{\circ} \mathrm{C}$. A long dry season of about 7-8 months is responsible for the occurrence of tropical dry deciduous forests principally dominated by Tectona grandis with associates like Terminalia tomentosa W.\& A., Diospyros melanoxylon Roxb. and Butea monosperma Lamk.Taub. Soil is thin, rich in calcium with $\mathrm{pH}$ ranging from 6.5 to 8.5 .

\section{Materials and methods}

\section{Sample collection}

Soil and root samples were collected during the month June (Late Summer) from selected dominant and codominant forest tree species of all the three sites. Individual non-adhering rhizosphere soil samples were taken in triplicate from the depth of 10-15 $\mathrm{cm}$, after removing upper detritus. Fine rooflets (feedar roots) were collected from the dominant species of each sites. Samples were air dried, sieved and stored at $4^{\circ} \mathrm{C}$ until further processing. 


\section{Processing of root samples}

Root samples were rinsed with tap water and cut into $1 \mathrm{~cm}$ pieces, and the staining was done with trypan blue (Phillips and Haymann, 1970; Kormainik et al., 1980). One hundred root pieces were randomly picked and mounted on glass slides in lactophenol and examined under compound microscope. The colonization was calculated using the following formula

$$
\% \text { colonization }=\frac{\text { no. of root segments colonized }}{\text { Total no. of root segments studied }} \times 100
$$

Qualitative characteristics of mycorrhizal fungi i.e. external hyphae, presence of vesicles, Paris coil and occurrence of intraradical spores, were observed in stained root samples.

\section{Extraction of spores and their identification}

Spores from the soil samples $(25 \mathrm{~g})$ were extracted by wet sieving and decanting method (Gerdemann and Nicolson, 1963). For the estimation of VAM species, a modified method (Gour and Adholeya, 1994) was followed and identification was done on current descriptions and identification manuals (Schenk and Perez, 1990; Mehrotra and Baizal, 1994; www.invam.caf.wvu.edu/my-info/Taxonomy/species.htm). Species name code of VAM fungi were followed (Perez and Schenck, 1990).

\section{Results}

Data of rhizospheric soil analysis of three study sites is presented in the table 1 to 4 . Twelve tree species were analyzed for quantitative and qualitative characters of VAM fungi from site I. The lowest root colonization was observed in Walsura robusta Roxburgh (19\%) and highest in Terminalia myrocarpa Henrck \& Mull. (33\%) followed by Terminalia nodiflora (R. Ar.) (32\%) and Dillenia indica Linn. (32\%). VAM fungal spore population per $25 \mathrm{~g}$ of rhizospheric soil of 12 tree species from site I is presented in the Table 1. It is evident that the lowest spore population (48/25 gm) was observed in rhizosphere soil of Mesua ferrea Linn.and highest spore population (133/25 g) in Terminalia myrocarpa Henrck \& Mull. Qualitative characters of VAM fungi viz., external hyphae, vesicle, Paris coil, intraradical spores are given in Tables 1 to 3. The presence of extrametrical hyphae were recorded in Terminalia myrocarpa Henrck \& Mull., Chukrasia tabularis (A. Juss.), Tetrameles nodiflora ( R. Ar.), Meusa ferrea Linn., Artocarpus lakoocha Roxb.and Walsura robusta Roxb.. Vesicles were less common although observed in Dillenia indica Linn., Mesua ferrea Linn., Artocarpus lakoocha Roxb. and Walsura robusta Roxb. Paris type coils were observed in root pieces of Dipterocarpus macrocarpus Vesque, Castanopsis spp Blume., Chakrasia tabularis(A. Juss.), Terminalia myrocarpa Henrck \& Mull. and Artocarpus chama Buch.Ham. Intraradical spores were abundant with the exception of Dipterocarpus macrocarpus Vesque, Dillenia indica Linn., Artocarpus lakoocha Roxb. and Artocarpus chama Buch.Ham. Total 15 VAM species were recovered from this site, out of which 5 VAM species were exclusive, i.e. restricted to this site only. 
Table 1. Quantitative and Qualitative characters of VAM fungi associated with some important forest tree species at Site-I (Jorhat)

\begin{tabular}{|c|c|c|c|c|c|c|c|c|c|c|c|c|}
\hline 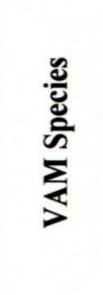 & 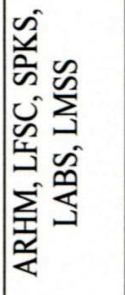 & 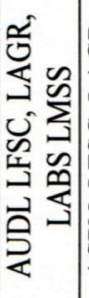 & 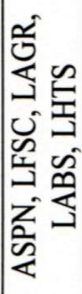 & 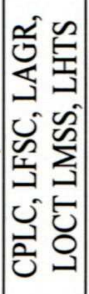 & 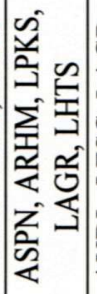 & 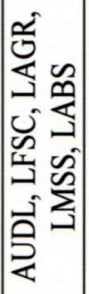 & 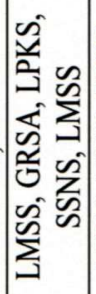 & 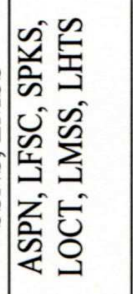 & 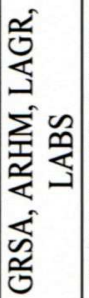 & 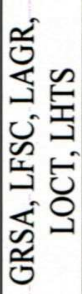 & 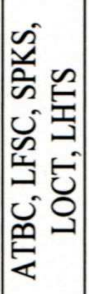 & 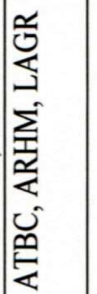 \\
\hline 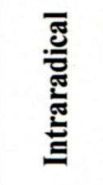 & ' & + & ' & + & + & + & + & + & + & ' & ' & + \\
\hline 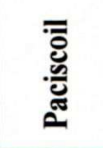 & + & + & ' & ' & ' & + & ' & 丰 & ' & . & + & ' \\
\hline $\begin{array}{l}\frac{0}{\frac{L}{2}} \\
\frac{0}{y}\end{array}$ & ' & ' & $\ddagger$ & ' & ' & ' & ' & ' & + & + & ' & + \\
\hline 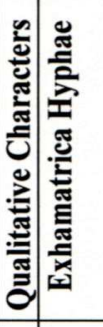 & ' & ' & ' & ' & + & + & ' & + & + & + & ' & + \\
\hline 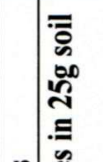 & 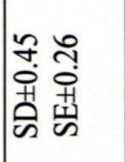 & 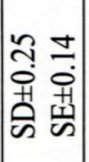 & 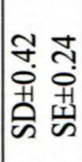 & $\begin{array}{l}\sqrt{\tilde{0}} \\
\text { 㺃 } \\
\text { के }\end{array}$ & 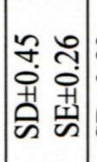 & 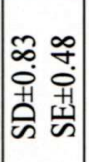 & $\begin{array}{cc}\infty & 0 \\
0 & \vdots \\
0 & 0 \\
1 & 4 \\
0 & 4\end{array}$ & 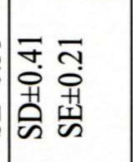 & 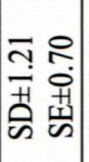 & 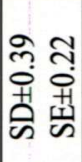 & 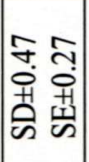 & 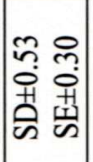 \\
\hline 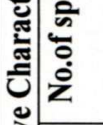 & $\infty$ & $\because$ & $\approx$ & $\cong$ & J & $\widetilde{\Xi}$ & 2 & $\cong$ & $\infty$ & 2 & $\Xi$ & in \\
\hline 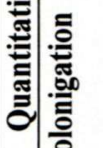 & 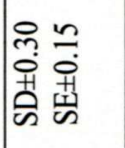 & 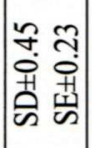 & 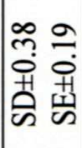 & 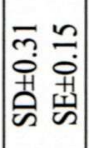 & 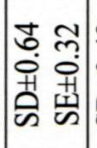 & 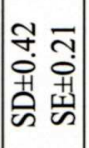 & 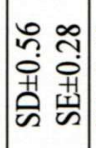 & 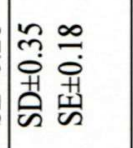 & 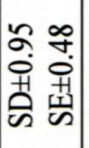 & 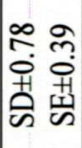 & 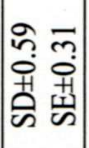 & 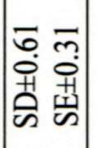 \\
\hline 递 & নి & $\approx$ & लె & $\approx$ & $\approx$ & 유 & $\stackrel{\sim}{\infty}$ & $m$ & $\bar{N}$ & $\approx$ & প্ল & 2 \\
\hline 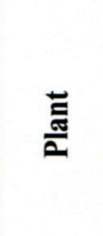 & 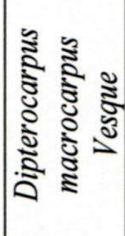 & 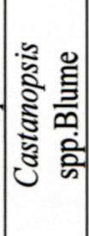 & 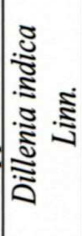 & 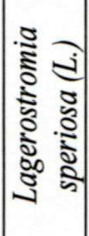 & 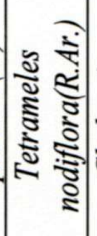 & 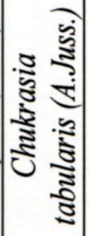 & 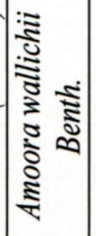 & 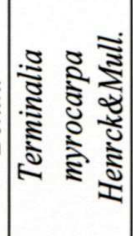 & 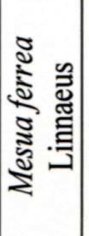 & 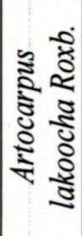 & 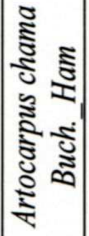 & 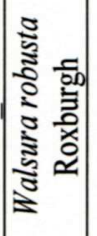 \\
\hline$\dot{n} \dot{z}$ & - & $N$ & $m$ & $\theta$ & in & 0 & $r$ & $\infty$ & $a$ & 으 & $=$ & $\simeq$ \\
\hline
\end{tabular}


Table 2. Quantitative and Qualitative characters of VAM fungi associated with some important forest tree species at Site-II (Mandla)

\begin{tabular}{|c|c|c|c|c|c|c|c|c|c|c|c|c|c|c|}
\hline 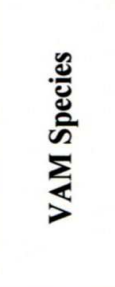 & 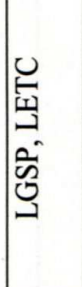 & 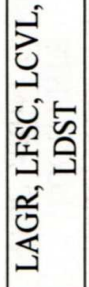 & 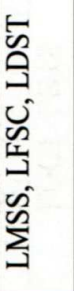 & 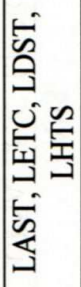 & 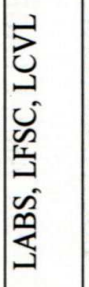 & 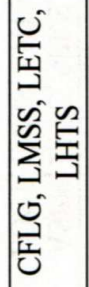 & 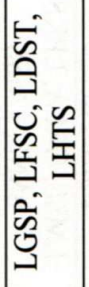 & 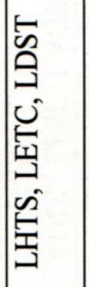 & 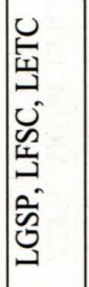 & 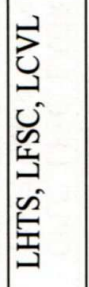 & 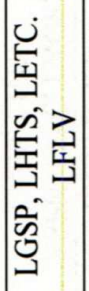 & 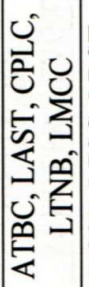 & 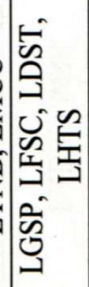 & 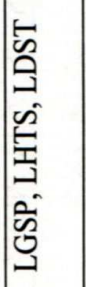 \\
\hline 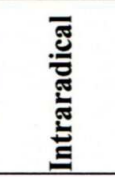 & + & + & + & ' & ' & ' & + & + & ' & + & & ' & + & ' \\
\hline 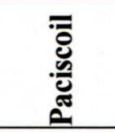 & + & + & ' & + & + & + & ' & + & + & + & + & + & + & ' \\
\hline$\frac{\stackrel{0}{\frac{D}{5}}}{2}$ & ' & + & + & ' & + & + & + & ' & + & ' & + & + & + & + \\
\hline 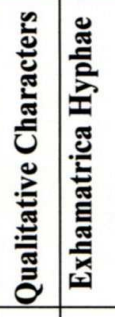 & + & + & + & + & + & + & ' & + & ' & ' & ' & $\begin{array}{l}+ \\
+ \\
+\end{array}$ & + & \\
\hline \multirow{4}{*}{ 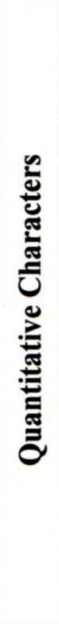 } & 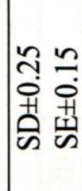 & 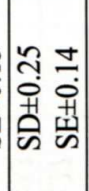 & 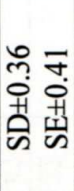 & 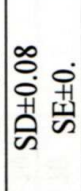 & 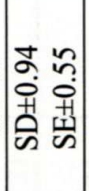 & $\begin{array}{ll}\infty & 0 \\
0 & 0 \\
0 & 0 \\
0 & 0 \\
0 & 4\end{array}$ & 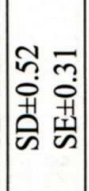 & 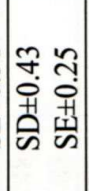 & 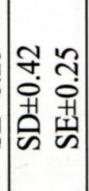 & 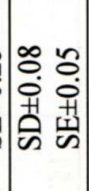 & 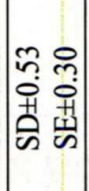 & 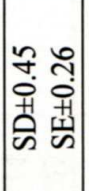 & $\begin{array}{l}0 \\
0 \\
0 \\
0 \\
0 \\
0\end{array}$ & 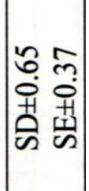 \\
\hline & F & 2 & \$ & $\tilde{\varrho}$ & $\bar{a}$ & $\widehat{\Xi}$ & in & $\infty$ & $\stackrel{m}{m}$ & 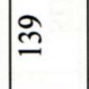 & $\cong$ & 16 & $\bar{a}$ & ¿ \\
\hline & $\begin{array}{l}\text { f. } \\
\text { 号 } \\
\text { 葸 } \\
\text { की }\end{array}$ & 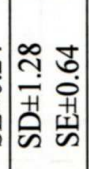 & 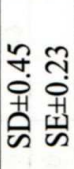 & 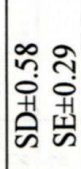 & 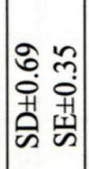 & 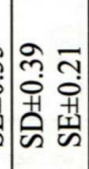 & 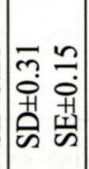 & 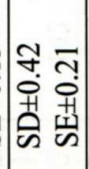 & 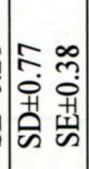 & 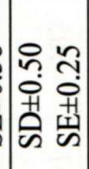 & 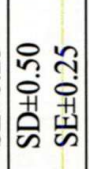 & 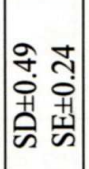 & $\mid \begin{array}{l}2 \\
0 \\
0 \\
0 \\
0\end{array}$ & 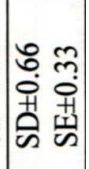 \\
\hline & $\Re$ & $\infty$ & $q$ & $F$ & $\approx$ & $\approx n$ & 8 & i & in & ले & 웅 & J & $\Re$ & 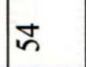 \\
\hline $\begin{array}{l}\frac{\vec{E}}{\alpha} \\
\frac{\mathrm{E}}{\alpha}\end{array}$ & 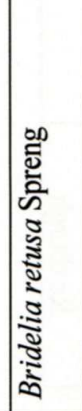 & 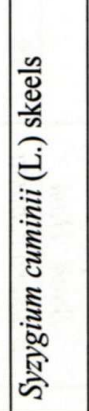 & 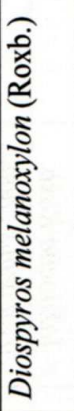 & 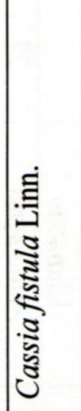 & 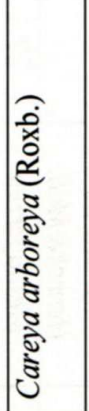 & 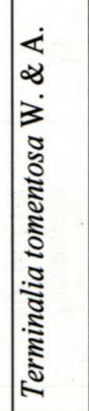 & 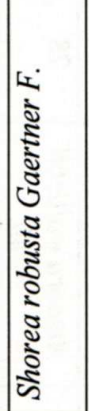 & 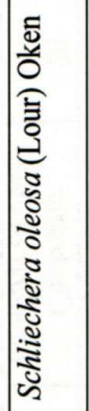 & 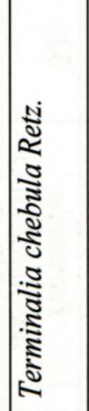 & 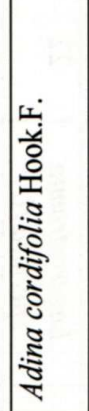 & 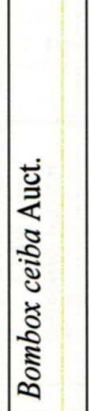 & 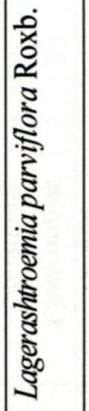 & 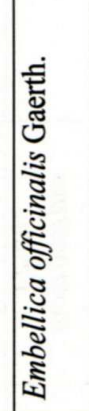 & 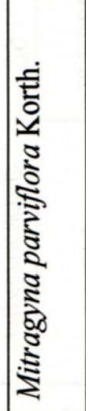 \\
\hline$\dot{\dot{n}} \dot{z}$ & - & N & $m$ & $\nabla$ & in & 6 & n & $\infty$ & $a$ & 으 & $=$ & $\simeq$ & $\cong$ & \pm \\
\hline
\end{tabular}


Table 3. Quantitative and Qualitative characters of VAM fungi associated with some important forest tree species at Site-III (Sagar)

\begin{tabular}{|c|c|c|c|c|c|c|c|c|c|c|c|c|c|}
\hline \multicolumn{2}{|l|}{ 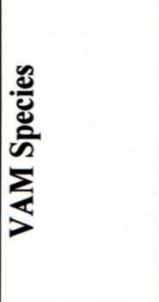 } & 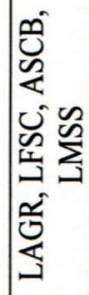 & 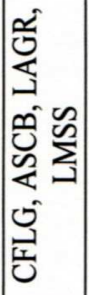 & 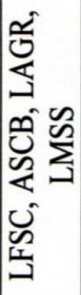 & 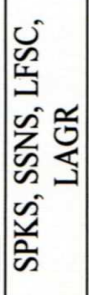 & 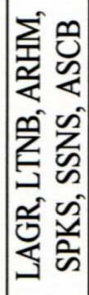 & 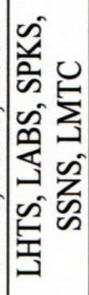 & 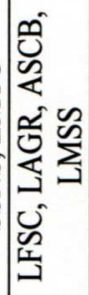 & 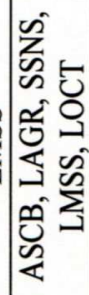 & 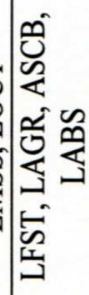 & 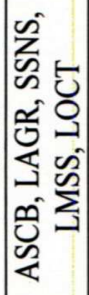 & 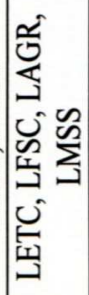 & 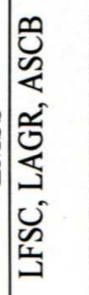 \\
\hline \multicolumn{2}{|l|}{ 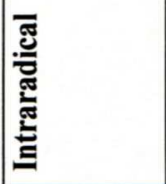 } & + & + & ' & ' & + & + & + & + & ' & ' & ' & + \\
\hline \multicolumn{2}{|l|}{ 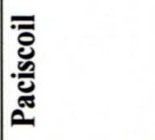 } & + & + & ' & ' & + & + & ' & $\stackrel{+}{+}$ & ' & ' & ' & ' \\
\hline \multicolumn{2}{|l|}{$\frac{\pi}{\frac{\pi}{2}}$} & + & + & + & + & $\begin{array}{l}+ \\
+\end{array}$ & + & + & + & + & + & ' & ' \\
\hline 起 & 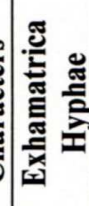 & + & ' & ' & ' & ' & + & ' & ' & + & + & $\stackrel{+}{+}$ & ' \\
\hline \multirow{4}{*}{ 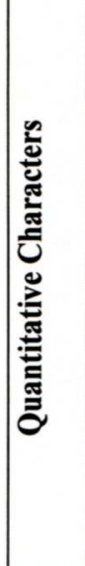 } & \multirow{2}{*}{ 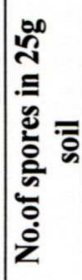 } & 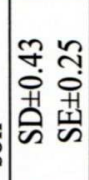 & 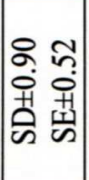 & 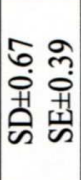 & 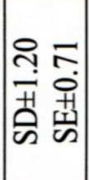 & 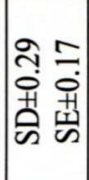 & 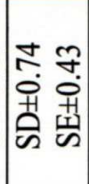 & 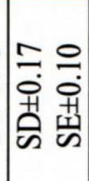 & $\begin{array}{l}m \text { n } \\
\text { 章 } \\
\text { 䓵 }\end{array}$ & 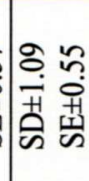 & 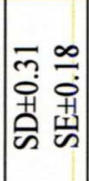 & 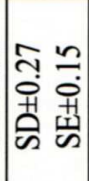 & $\begin{array}{l}n \\
\text { ñ } \\
0 \\
0 \\
0 \\
0 \\
\text { की }\end{array}$ \\
\hline & & $F$ & $\mathbb{J}$ & సิ & 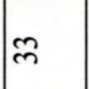 & 2 & $\tilde{\varrho}$ & 5 & $\Xi$ & $\bowtie$ & $\bar{\beth}$ & $\approx$ & sa \\
\hline & \multirow[t]{2}{*}{ 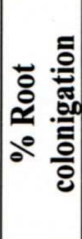 } & 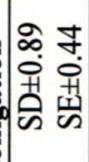 & 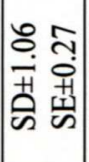 & 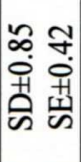 & 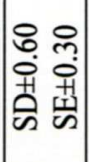 & 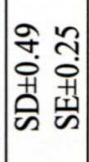 & 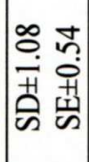 & 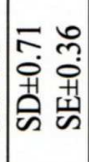 & 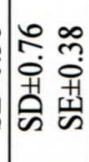 & 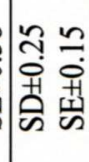 & 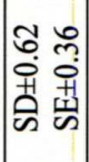 & 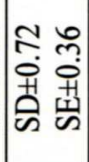 & 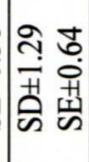 \\
\hline & & 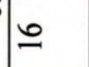 & নి & \pm & $\bar{\sim}$ & $\underset{n}{\infty}$ & 点 & $\approx$ & $\stackrel{\infty}{n}$ & 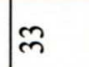 & สิ & $\nabla$ & $\Re$ \\
\hline \multicolumn{2}{|l|}{ 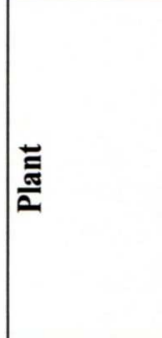 } & 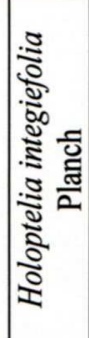 & 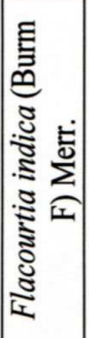 & 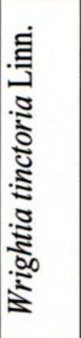 & 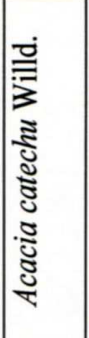 & 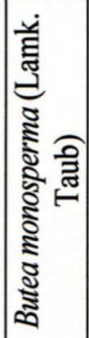 & 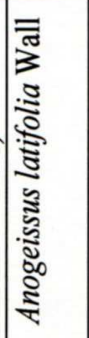 & 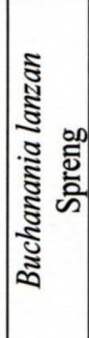 & 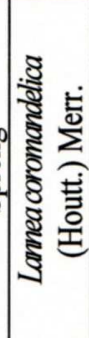 & 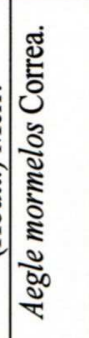 & 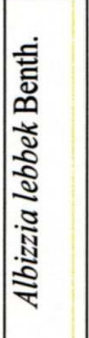 & 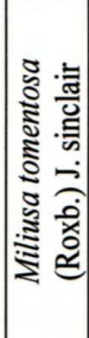 & 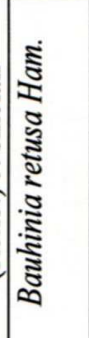 \\
\hline \multicolumn{2}{|c|}{$\dot{n} \dot{z}$} & - & $\sim$ & $m$ & $\nabla$ & in & 0 & r & $\infty$ & $a$ & 으 & $=$ & $\simeq$ \\
\hline
\end{tabular}


Table 4. Occurence of different VAM Species at three different forest vegetation

\begin{tabular}{c|c|c}
\hline Jorhat (site-I) & Mandla (Site-II) & Sagar (Site-III) \\
\hline LFSC $^{*}$, LABS*, LMSS*, & LFSC*, LCVL, LGSP, & LAGR*, LFSC*, LMSS*, \\
LHTS* & LAGR* & LABS* \\
LAGR*, LOCT, LMST, & LETC, LMSS*, LDST, LAST & LTNB, LHTS*, LMTS, LOCT \\
ARHM & & \\
AUDL, ASPN, ATBC, SPKS & LHTS*, LABS*, LTNB, & LETC, ASCB, ARHM, CFLG \\
LMNS, CPLC, GRSC & CFLG, CPLC, ATBC, LFLV & SPKS, SSNS \\
\hline
\end{tabular}

*= common species, \# =exclusive species

- species code of VAM fungi were followed after Perez and Schenk (1990)

$\mathrm{LABS}=\mathrm{G}$. ambisoporum smith and schenck.

$\mathrm{LTNB}=\mathrm{G}$. tenebrosum(Thaxter) Berch.

LHTS $=$ G. heterosporum Smith and shenck.

$\mathrm{LMST}=\mathrm{G}$. multisubtensum mukerji,

Bhattacharjee and Tiwari.

$\mathrm{LOCT}=\mathrm{G}$. occultum Walker.

LETC $=$ G. etunicatum Becker and Gerdemann.

$\mathrm{LCVL}=\mathrm{G}$. convolutum Gerdemann and Trappe.

$\mathrm{LGSP}=\mathrm{G}$. geosporum (Nical and Gerd.) walber.

LDST $=$ G. deserticola Trappe, Bloss and Menga.

LAST $=$ G. australe (Berk) Berch.

$\mathrm{LMCC}=\mathrm{G}$. macrocarpum Tulasne and Tulasne.

LPST $=$ G. postulatum Koske, friese. walker and Dalpe.

LMTC $=$ G. multicaule Gerdemann and Bakshi.

$\mathrm{ASCB}=$ Acaulospora Scrobiculata Trappe.

$\mathrm{ARHM}=\mathrm{A}$. rehmii sieverding and Toro.

LAGR $=$ Glomus aggregatum schenck and smith emend. koske.

LFSC $=$ Glomus fasciculatum (Thaxter) Gerdemann and Trappe emend. walker and koshe.

LMSS $=$ G. moseae(Nicolson and Gerdemann) Gerdemann and Trappe.

At site II, 14 tree species were selected for analysis of different attributes. Lowest root colonization was observed in Bridelia retusa Spreng. (33\%) and highest $(64 \%)$ in Lagerastroemia parviflora Roxb. followed by Terminalia chebula Retz. (57\%). VAM fungal spore population per $25 \mathrm{~g}$ of soil collected from the rhizosphere of 14 tree species (Table 2) showed wide variations from 43 in Bridelia retusa Spreng to 167 in Lagerastroemia parviflora Roxb.

Data indicate that extramatrical hyphae were present in all the tree species except in Terminalia chebula Retz., Adina cordifolia Hook.F., Bombox ceiba Auct. and Mitragyna parviflora Korth.. Occurrence of vesicles was common however, not observed in Bridelia retusa Spreng., Cassia fistula Linn., Schliechera oleosa (Lour) Oken. and Adina cordifolia Hook. F.. Paris coil type association was a common feature in all the tree species barring Diospyros melanoxylon (Roxb.), Shorea robusta Gaertner F. and Mitragyna parviflora Korth.. Intraradical spores were found in Bridelia retusa Spreng, Syzygium cuminii (L.)Skeels, Disopyros melanoxylon (Roxb.) Shorea robusta Gaertner F., Schliechera oleosa(Lour) Oken, Adina cordifolia Hook. F. and Embellica officinalis Gaerth.

Total 16 VAM species were found at the site II, 6 species were exclusive to this site.

From site III, twelve tree species were considered and root colonization was minimum in Wrightia tinctoria Linn. (14\%) and maximum (62\%) in Albizzia lebbek Benth. followed by Lannea coromandelica (Houtt.) Merr. (58\%). VAM fungal spore population per $25 \mathrm{~g}$ of rhizospheric soil for 12 selected tree species (Table 3 ) \& showed 
highest spore population (121/25 g) in Albizzia lebbek Benth.and lowest (29/25 g) in Wrightia tinctoria Linn.

External Hyphae were present in Holoptelia integrifolia Planch and Anogeissus latifolia Wall. Vesicles were found in 10 tree species except in Miliusa tomentosa and Bauhinia retusa. Paris type coils were recorded in the Holoptelia integrifolia Planch, Flacoutia indica (Burm F) Merr., Butea monosperma (Lamk.Taub.), Anogeissus latifolia Wall, Lannea coromandelica (Houtt.) Merr.. Intraradical spores were found within most of the tree species except in Wrightia tinctonia Linn., Acacia catechu Willd, Aegle marmelos Correa, Albizzia lebbek Benth., Miliusa tomentosa (Roxb.) J. Sinclair.

Total 14 VAM species were recorded from site III in which 2 VAM species were exclusive.

Total 25 VAM species were observed from all the three study sites. Out of which 15 were from site I, 16 from site II and 14 VAM species from site III (Table 4). 5 VAM species were common to all the three sites.

\section{Discussion}

Arbuscular mycorrhizal fungi are most widely distributed symbiotic organisms in nature. The soil borne mycorrhizal fungi colonize the root cortex biotropically followed by the development of external mycelium which is a bridge connecting the root with the surrounding soil microhabitats. The VAM fungi have been reported from different plant communities of varied geographical regions such as - Tropical region (Chaurasia and Khare, 2000); Temperate region (Vestberg, 1995) and arctic region (Dalpe and Aiken, 1998).

Results of the present study indicate that all the tree species from tropical forests had VAM association with variation in the degree of infection. Such variability of colonization has been observed by number of workers in tropical forests and attributed to a number of local edaphic and climate factors (Safir and Dunisway, 1982; Thapar and Dunisway, 1992; Byra Reddy et al., 1994; Rahangdale and Gupta, 1998; Kalita et al., 2004).

Highest spore population was observed in rhizsophere soils of site II than at site I and site III. These differences can be attributed to the type of VAM species associated with their plants (Pringle and Bever, 2002). Seasonal variation in spores population in tropical forests depend upon the climatic factors, soil type, nutrients status growth and metabolic activities of plants, together with preferences of the fungi to host species and spatial distribution of host species (Louis and Lim, 1997; Harinikumar and Bagyaraj, 1988; Johnson and Wedin, 1997; Allen et al., 1998; Lovelock et al., 2003). Moreover, VAM spore densities vary greatly according to the physiological state of the host plant and relationship with the plant species. It is interesting that Arbuscules (Arum type) in VAM fungi were absent from all the three sites, although as a defining characteristic, arbuscules may be difficult to find under natural conditions due to their short life span (Smith and Read, 1997).

Paris type (Hyphal coil) association were more frequently observed. They were particularly prolific at the site II than in the other two sites. The dominance of Paris type association imply that coiled hyphae live longer and are more tolerent to stress conditions such as drought, suggesting that external factors determine the dominance of the type of endomycorrhizae (paris Vs arum) over another. 
The frequent occurrence of vesicles in most of the tree species from all the three study sites showed the presence of VAM fungi belonging to the Glomineae. The highest frequency of oval to elongate vesicles over the irregular and lobbed vesicles showed the dominance of species of the genus Glomus among other genera. Predominance of Glomineae has already been reported from tropical sites (Sieverding, 1991; Onguene and Kuyper, 2001). Results also showed the dominance of Glomineae over Gigasporineae. In the present study, the rhizosphere of different forest tree species from three forest sites showed common as well as variant VAM flora. Such variations are common features of VAM (Klironomos et al., 1993; Vishalakshi, 1997; Rawat and Mukerjii, 1998; Muthukumar and Udaiyan, 2000; Rama Bhat and Kaveriappa, 2001; Tamuli and Boruah, 2002).

A large number of VAM speices belong to the genus Glomus in the present study. The genus Glomus has been reported to be the dominant VAM fungus in a number of forest communities (Sharma et al., 1986; Byra Reddy et al., 1994; Uniyal and Uniyal, 2000; Tamuli and Boruah, 2002; Mohan Kumar and Sivaswamy, 1992). This may be explained due to the wider adaptability of Glomus spp. as compared to other species (Sparling and Tinker, 1978; Sharma et al., 1986; Byra Reddy et al., 1994; Schenk and Kinloch, 1980).

Comparative assessment of occurrence of VAM fungi from three sites envisage that tropical moist forests (site II) harbour more VAM population in terms of richness and diversity than tropical evergreen and deciduous forest communities.

Acknowledgements. Author wish to thank Dr. P. K. Khatri, Tropical Rain Forest Institute, and Dr. R.K. Shukla, Kanha Tiger reserve, Mandla, for providing field facility. One of the authors (Priyangana Tripathi) is thankful to University Grants Commission, New Delhi, for the award of fellowship and financial assistance.

\section{REFERENCES}

[1] Allen, E.B., Rincon, E., Allen, M.F., Perez-Jimenez, A., Haunte, P. (1998): Distribution and Seasonal dynamics of mycorrhizae in a tropical deciduous forest in Mexico. Biotropica 30: 261-274.

[2] Bhat, M.N. (1993): Vesicular-arbuscular mycorrhizae in submouthain region. - Acta Bot. Indica 25: 52-56.

[3] Byra Reddy, M.S., Krishna, L., Naik, Bhaskar, V., Bagyaraj, D.J. (1994): Status of mycorrhizal associations in some tropical tree species. - J.Soil Biol. And Ecol.14: 51-57.

[4] Champion, H.G., Seth, S.K. (1968): A revised survey of the forest types of India. - Govt. of India Publication, New Delhi, pp.404.

[5] Chaurasia, B., Khare, P.K. (2000): Ecological study of tropical forest trees with special reference to vesicular Arbuscular Mycorrhizal(VAM) Association.

[6] Chaurasia, B., Khare, P.K. (2005): Hordeum vulgare: a suitable host for mass production of arbuscular mycorrhizal fungi from natural soil. - Appl. Environ. Res. 4: 45-53.

[7] Dalpe, Y., Aiken, S.G. (1998): Arbuscular mycorrhizal fungi associated with festuca species in the Canadian High Arctic. - Can. J. Bot. 76: 1930-1938.

[8] Gerdemann, J.W., Nicolson, T.H. (1963): Spores of mycorrhizal Endogone species extracted from soil by wet sieving and decanting. - Trans. Br. Mycol. Soc. 46: 235-244.

[9] Gour, A., Adholeya, A. (1994): Estimation of VAMF Spores in soil: A modified method. - Mycorrhiza news 6: 10-11. 
[10] Harinikumar, K.M. Bagyaraj, D.J. (1988): The effect of season on a VA mycorrhizae of Leucaena and Mango in a semi-arid tropic Arid Soil Res. - Rehabil. 2: 139-143.

[11] Hodge, A., Campbell, C.D., Fitter, A.H. (2001): An arbuscular mycorrhizal fungus accelerates decomposition and acquires nitrogen directly from organic material. - Nature 413: 297-299.

[12] Johnson, N.C., Wedin, D.A. (1997): Soil carbon nutrients and mycorrhizae during conversion of dry tropical forest to grassland. - Ecol. Appl. 7: 171-182.

[13] Kalita, R.K., Dutta D., Borah, D.P. (2004): Association of arbuscular mycorrhizas with twenty biomass species in Assam. - Indian forester: 699-703.

[14] Klironomos, J.N., Moutoglis, P., Kendrick, B., Widden, P. (1993): A comparison of spatial heterogeneity of Vesicular Arbuscular Mycorrhizal fungi in two maple forest soil. - Can. J. Bot. 71: 1472-1480.

[15] Kormainik, P.P., Bryan, W.C., Shulz, R.C. (1980): Procedure and equipment for staining large number of plant roots for endomycorrhizal assay. - Can. J. Microbiol. 26: 536-538.

[16] Louis, I., Lim, G. (1997): Spore density and root colonization of vesicular arbusculas mycorrhizar in tropical soil. - Trans. Br. Mycol. Soc. 88: 207-212.

[17] Lovelock, D.E., Anderson, K., Morton, J.B. (2003): Arbuscular mycorrhizal communities in tropical forests are affected by host tree species and environment. - Oecologia 135: 268-279.

[18] Mehrotra, V.S., Baizal, U. (1994): Advances in the taxonomy of Vesicular arbuscular mycorrhizal fungi. - Biotechnology in India: 227-286.

[19] Mohan Kumar, V., Sivaswamy, S.N. (1992): Vesicular abuscular mycorrhizae Adv. - Pl. Sci. 50: 17-28.

[20] Muthukumar, T., Udaiyan, K. (2000): Arbuscular Mycorrhizas of plants growing in western ghats. South India. - Mycorrhiza 9: 297-313.

[21] Onguene, N.A., Kuyper, T.W. (2001): Mycorrizal association in the rain forest of South Cameroon. - For. Ecol. Mange 140: 277-287.

[22] Pate, J.S. (1994): The Mycorrhizal association: Just one of many nutrient acquiring specialization in natural ecosystems. - In: Robson, A.D., Abbott, L.K., Malajezuk, N. (Eds). Management of Mycorrhizas in Agriculture and Forestry. Kluwer Academic Publishers, London, 1-10.

[23] Perez, Y., Schenck, N.C. (1990): A unique code for species of VAM mycorrhizal fungi. Mycologia 82: 256-260.

[24] Phillips, J.M., Haymann, D.S. (1970): Improved procedure for cleaning roots and staining parasitic and vesicular arbuscular mycorrhizal fungi for rapid assessment of infections Trans. - Br. Mycol. Soc. 55: 158-161.

[25] Pringle, A., Bever, J.D. (2002): Divergent phenologies may facilitate the coexistence of arbuscular mycorrhizal fungi in a North Carolina Grassland. - An. J. Bot. 89: 1439-1446.

[26] Rausch, C., Daram, P., Brunner, S. Jansa, J., Lalol, M., Leggewele, G., Amrhein, N., Bucher, M. (2001): A phosphate transporter expressed in anbuscule-containing cells in potato. - Nature 414: 462-466.

[27] Rahangdale, R., Gupta, N. (1998): Selection of some VAM inoculants for some forest tree species. - Indian Forester 124(5): 31-341.

[28] Rama Bhat, P., Kaveriappa, K.M. (2001): Population of Arbuscular Mycorrhizae (AM) of Myristica fatua var. magnifica in ex-situ conditions. Frontier in fungal biotechnology anf plant pathogens. - Proc. Intl. conference ( Mnoharachary, C. ed.) Osmania University, Hyderabad, 299-308.

[29] Rawat, R., Mukerjii, K.G. (1998): Vesicular Arbuscular Mycorrhyzal Association in sugarcane. - Phytomorph 48: 309-316.

[30] Safir, G.R., Dunisway, J.M. (1982): Evaluation of plant response to colonization by vesicular arbuscular mycorrhizal fungi. Methods and Principles of Mycorrhizal Research (N.C.Schenck,ed.). - American Phytopathological Society, St. Paul, Minnesota, USA: 7780 .

APPLIED ECOLOGY AND ENVIRONMENTAL RESEARCH 10(4): 561-571. http://www.ecology.uni-corvinus.hu • ISSN 15891623 (Print) • ISSN 17850037 (Online) (C) 2012, ALÖKI Kft., Budapest, Hungary 
[31] Schenk, N.C., Perez, Y. (1990): Manual for the identification of VA mycorrhizal fungi. Synergetics Publication, Gainesville, Florida.

[32] Schenk, N.C., Kinloch, R.A. (1980): Incidence of mycorrhizal fungi on six field crops in monoculture on a newly cleared woodland site. - Mycologia 72: 445-455.

[33] See www.invam.caf.wvu.edu/my-info/Taxonomy/species.htm/.

[34] Sharma, S.K., Sharma, G.D., Mishra, R.R. (1986): Status of mycorrhizae in sub-tropical forest ecosystem of Mehalaya. - Acta Bot. Indica 14: 87-92.

[35] Sieverding, E. (1991): Vesicular arbuscular mycorrhizal management in tropical agrosystems. - Deutsche Gesellschaft fur Technische Zesammenarbeit(GTZ), Eschborn, Germany.

[36] Smith, S.E., Read, D.J. (1997): Mycorrhizal Symbiosis. - $2^{\text {nd }}$ Ed. Academic Press, London.

[37] Sparling, G.P., Tinker, P.B. (1978): Mycorrhizas in Pennie grassland. I. Level of Infection in the field. - J. Appl. Ecol. 15: 943-950.

[38] Tamuli, P., Boruah, P. (2002): VAM association of Agar wood tree in Jorhat district of the Brahmaputra Valley. - Indian Forester 128(9): 991-994.

[39] Taylor, T.N., Remy, W., Hass, H., Kerp., H. (1995): Fossil arbuscular mycorrhizae from the early Devonian. - Mycologia 87: 560-573.

[40] Thapar, G.R., Dunisway, J.M. (1992): Evaluation of plant response to colonization by vesicular arbuscular mycorrhizal fungi. Methods and principles of Mycorrhizal Research (N.C.Schenck, ed.). - American Phytopathological Society. St.Paul, Minnesota, USA: 7780.

[41] Uniyal, K., Uniyal, D.P. (2000): Populatio dynamics of arbuscular mycorrhizal fungi in Dalbergia sisso. Roxb. - Indian Forester 126(7): 782-787.

[42] Vestberg, M. (1995): Occurrence of some Glomales in Finland. Mycorrhiza.S 5: 329-336.

[43] Vishalakshi, N. (1997): Dynamics of vesicular arbuscular mycorrhizae in two tropical dry evergreen forest, South India. - Int. J. Ecol. Environ. Sci. 23:25-36. 